\title{
REDUCTION OF MIXING IN JET-FED WATER STORAGE TANKS
}

\author{
D.B. Martinsen and A.D. Lucey \\ School of Engineering, University of Warwick \\ Coventry, CV4 7AL, U.K. \\ Tel.: +44-(0)24-76523143, Fax.: +44-(0)24-76418922, E-mail: tony@eng.warwick.ac.uk
}

\begin{abstract}
Contrary to usual mains-water practice, mixing in water storage tanks used in rainwater harvesting systems is undesirable because pathogen die-off can occur in the unmixed water prior to its extraction for use. The principal cause of mixing in these tanks is the momentum of the inflow during a rainfall event. We investigate the effect of inflow-jet configuration on the proportion of stored water in a tank which mixes with the slightly cooler inflow of rooftop water. Scale experiments are conducted which show that the non-dimensional height of the mixing front above the jet inlet is proportional to the inflow-jet densimetric Froude number for both single and multi-jet arrangements of various geometries. For each arrangement a coefficient of mixing is found. The results are then used to assess the level of mixing in full-scale rooftop rainwater harvesting storage tanks and determine whether residence time in such tanks is a viable strategy for pathogen reduction. For such applications, a radial manifold of jets outwardly directed from the tank centre is found to be the most promising.
\end{abstract}

\section{INTRODUCTION}

The collection of rainwater from dwelling roofs and its storage in householders' tanks is a means of water supply which is particularly appropriate in developing countries. The principal advantages of this type of rainwater harvesting are its relative inexpense and the decentralised nature of its management. However, one barrier to its more widespread use is the problem of water contamination arising from bacteria being washed into the supply from the roof. A possible way to combat this health hazard is to apply the common practice of reducing the pathogen count through storage (see, for example, Droste 1997). This technique could be especially cost-effective because a storage tank is already an integral part of a rainwater harvesting system. For such a strategy, water must be added to one part of the tank and taken from another. Moreover, to permit sufficent residence time for bacteria extinction in the outlet region of the tank's volume, it is essential that mixing of the entire tank's volume is minimised. In this paper we consider the inflow of water during a period of rainfall as the principal cause of mixing in the tank. Thus, we investigate a range of inflow-jet configurations, our goal being to identify that which causes the least mixing of the water already resident in the storage tank.

The objective of the present work is contrary to the usual practice in mains water systems wherein mixing is desireable as part of a chemical process. Enhanced mixing is also a feature of many chemical processes so as to reduce reaction times. Consequently much attention has been giving to strategies yielding increased mixing. Nevertheless, some work (for example, Davidson \& Adams 1994) has focused on reduced mixing in hotwater systems, notably through manifold inlet designs able to distribute water to an appropriate stratified layer according to its temperature. However, the present work draws most closely on the study of Rossman \& Grayman (1999). They experimentally investigated the mixing of water already resident in a container with that of a volume of cooler or warmer water added to it through a simple jet.
In particular, they found that the occurrence of complete mixing in the container depended upon an appropriate combination of its non-dimensional (with respect to jet diameter) depth and the densimetric Froude number based on the jet mean speed and reduced gravity. Moreover, the threshold between complete and partially mixed outcomes could be defined by a linear relationship between the two non-dimensional groups. In this paper we show that the relationship continues to hold where a mixed-water height replaces the tank depth. The mixedwater height is a measure of the original tank water which has been mixed with the inflow. Furthermore, we show that the linear relationship between the mixedwater height and Froude number continues to hold for a variety of inlet jet configurations with the value of the constant of proportionality being dependent upon the configuration. Our findings are then used to assess the suitability of storage as a strategy for bacteria reduction in domestic rainwater harvesting systems.

\section{MODEL OF WATER MOVEMENT IN RAIN- WATER TANKS}

Mixing in a rainwater tank occurs through a number of processes, the most important being diffusion, thermal convection and added momentum through an inlet. Diffusion is a slow process for the time-scales considered herein, requiring weeks for any significant movement. Thermal convection can be controlled by shading or burying the tank. This leaves inlet-water momentum as the principle driver of mixing in a rainwater storage tank.

The proposed strategy for bacteria reduction requires water collected from the roof to be fed into the bottom of the storage tank whilst the user draws water the top water through a floating outlet. Three notional zones exist within in the tank. From its base to a height $h$ is a mixing zone wherein water added through the inlet mixes with that already in the tank. Above this is an ageing zone, the water of which has been resident in the tank long enough since the previous rainfall event for viscous effects to have dissipated any residual circula- 
tion. With the addition of new rainwater to the mixing zone below, this zone moves, as a slug of fluid, upwards within the tank. Finally, above the ageing zone there is a freshwater zone from which water is drawn for use. Of course, the water here comes from the ageing zone; as water in the ageing zone moves up the tank with subsequent rainfall events, sufficient time passes for bacteria die-off to occur before it reaches the outlet. To permit enough time for this occur, it is essential that height of the interface between the mixing and ageing zones is minimised for a given addition of new water to the tank. This requirement motivates the present investigation of inlet-jet arrangements.

The stratification implied by the zonal categorisation above is supported by the fact that the rainwater entering through the inlet is at a lower temperature (typically $5^{\circ} \mathrm{C}$ ) than the water already in the tank. The dependence of water density, $\rho$ (in $\left.\mathrm{kg} / \mathrm{m}^{3}\right)$, on its temperature, $T$ (in degrees Celsius), for the range $15^{\circ}<T<45^{\circ}$ is approximately given by

$$
\rho=1000.3-0.005\left(T^{2}+T\right) .
$$

A reduced gravity, $g^{\prime}$ is then defined by

$$
g^{\prime}=g\left(\frac{\rho_{f}}{\rho_{t}}-1\right),
$$

where $\rho_{f}$ and $\rho_{t}$ are respectively the densities of the feed water and the ambient water in the tank. Following Fisher et al. (1979), we now define the densimetric Froude number, $F_{d}$, of the jet as

$$
F_{d}=\frac{u}{\sqrt{g^{\prime} d}}
$$

where $u$ is the mean speed of the jet fluid at entry to the tank and $d$ is the jet diameter. When a buoyant (here negatively) jet discharges into unconfined or semiconfined ambient water, there is a competition between any vertically directed momentum of the jet the buoyancy forces acting on it. Thus there exists a vertical distance from the jet's entry point for which further vertical travel ceases. Fisher et al. found this distance, $h$, to obey the equation

$$
\frac{h}{d}=k F_{d}
$$

where $k$ is a constant which lies in the range 0.5-0.7.

Rossman \& Grayman (1999) have shown that (4) also holds for vertical or horizontal jets issuing into a confined body of fluid where jet impact on the walls of the container and its redirection play a significant part in the mixing dynamics. In their work, $h$ represented the threshold depth for which full mixing occurred and $k$ lay in the range $0.8-1.5$. Here, we show that (4) also holds when $h$ represents (by height) the part of the original volume of container water which has mixed with the inflow; we term it the mixed-water height. It also holds for a wide range of inlet flows with each inlet configuration having a particular value for the coefficient of mixing, $k$. Thus, for the present application, wherein we wish to minimise $h$ for a given inflow, one objective is to determine which configuration yields the lowest value of $k$.

\section{EXPERIMENTAL METHOD}

Experiments have been conducted on a scale model using the apparatus depicted in Fig. 1. The different inlet arrangements investigated are shown schematically in Fig. 2. If the densimetric Froude number is matched between model, $)_{m}$, and prototype, $)_{p}$, then, provided (4) characterises the height, $h$, of the mixing front above the inlet, the experimental results can be applied to rainwater tanks through the scaling

$$
\frac{h_{m}}{d_{m}}=\frac{h_{p}}{d_{p}}
$$

In order to study the range of Froude numbers appropriate to the prototype inflow, we use salinity to generate the reduced gravity of the feed water, an approach often used in model experiments; see, for example, Linden (1999). This permits the use of higher jet speeds which has the advantage of reducing the mixing time-scales in the experiments before equilibrium is achieved. The tank is made of clear acrylic and has a diameter, $D$, of $200 \mathrm{~mm}$. We remark that $D$ does not appear in the scaling law (5), nor does the overall tank geometry figure in the mixing model. However, in the investigation that follows, the distance, $x$ (see Fig. 2a) of the jet orifice to the opposing wall of the tank is varied and only very weak dependence on this distance is found. For all the results presented below, the jet orifice (or manifold) was located at $0.1 H$ above the bottom of the tank. The apparatus had a moveable base allowing this distance to be changed (keeping the vertical location of the inlet fixed). However, experimentation showed only weak dependence on this parameter provided it remained close to the base of the tank. Of course, in a real application, it would be undesirable to place the inlet at the bottom of the tank since deposited silt would be stirred up and entrained by the inflow. Jet-orifice sizes and values of the reduced gravity are given in the legends of the results figures.

The experimental procedure is illustrated schematically in Fig. 3. To visualise the interface between tank water mixed with the feed water, dye is also mixed with the saline feed water. Diffusion time-scales for the dye were much longer than those of the experiment. Throughout the experiment, the height of the interface between dyed and clear water, $h_{d}(t)$, and the water-surface height, $h_{w}(t)$ are measured for increments of time $t$. At the start of the experiment (Fig. 3a), $t=0$ and the height of clear water is $h_{w}(0)$. In the mixing period (Fig. 3b), $0<t<t_{m}$, where $t_{m}$ is the total mixing time, both the interface and the surface height increase but $\dot{h}_{d}(t)>\dot{h}_{w}(t)$, where the dot notation indicates differentiation with respect to time. Once 
mixing is complete (Fig. 3c), $t>t_{m}$ and both the interface and surface height continue to increase but now $\dot{h}_{d}(t)=\dot{h}_{w}(t)$. This means that the vertical extent of clear water, $\left[h_{w}(t)-h_{d}(t)\right]$ remains constant, although it continues to move upwards as a slug of fluid due to the continued addition of feedwater. The volume of the original clear water in the tank which has mixed with the inflow water is quantified by the mixed-water height, $h$, which is given by

$$
h=h_{w}(0)-\underbrace{\left[h_{w}(t)-h_{d}(t)\right]}_{\text {constant for } t>t_{m}}
$$

Thus, the experiment is stopped at a time $t=t_{f}$ when the value of $\left[h_{w}(t)-h_{d}(t)\right]$ shows no further change, indicating that $t_{f}>t_{m}$ and that the mixing process is complete. The mean speed of the inflow jet for the run is then found using

$$
u=\left(\frac{D}{d}\right)^{2} \frac{h_{w}\left(t_{f}\right)-h_{w}(0)}{t_{f}} .
$$

Most experimental runs had $h_{w}(0)=100 \mathrm{~mm}$ although a number used $70 \mathrm{~mm}$ to confirm that there was insignificant dependence of the mixing results upon $h_{w}(0)$. Throughout, corrections for temperature effects were incorporated in the evaluation of the densimetric Froude number. In addition to recording quantitative results, the light sheet depicted in Fig. 1 permits qualitative understanding through observation of the jet's behaviour and the mixing process.

\section{RESULTS}

Results for the straight horizontal inlet shown in Fig. 2a are presented in Fig. 4. Here, and in the following Figs. 5, 6 and 7, we plot the non-dimensional mixedwater height, $h / d$, against the densimetric Froude number. In Fig. 4 are collected the results of experimental runs for different inlet-jet diameters, $d: 6.5-10.5 \mathrm{~mm}$, a range of reduced gravity, $g^{\prime}: 2.1-8.5 \mathrm{~m} / \mathrm{s}^{2}$ and different values of the distance between the jet orifice and the opposing wall of the tank, $x: 10-190 \mathrm{~mm}$. Experimental observations show that, for low Froude numbers and high $x$, the jet first falls to the floor of the tank as it travels across to impact upon the far wall. For high Froude numbers or low values of $x$, the jet impacts directly upon the opposite wall. Thereafter, in both cases, the jet climbs the wall until it is overturned by the buoyancy forces to then spread out horizontally. Quantitatively, the present combinations of parameters show non-dimensional data collapse which follows the mixing rule (4) proposed by Fisher et al. (1979). The gradient of the best fit line drawn in Fig. 4, gives a coefficient of mixing, $k=0.91$, which is good agreement with the findings of Rossman \& Grayman (1999).

A horizontal circulating inlet (Fig. 2b) provided a spreading jet which spiralled downwards around the tank circumference to the bottom of the tank. Results for this case are plotted in Fig. 5. The shaded region in this and subsequent figures represents the area above the line of best-fit in the standard straight-inlet case of Fig. 4. Given the objectives of the present application, this inlet configuration, which has a measured coefficient of mixing of 0.65 , represents an improvement to the standard straight inlet.

A means of increasing inlet area and thus reducing jet velocity is to use a manifold inlet design. Each jet will have a Froude number and associated mixing height. However the total mixing height might be expected to be less than for a single jet of the same total cross-sectional area because each individual jet has a much smaller diameter. The characterisation of the manifold considered the effects of each jet in the manifold individually with the discharge rate being the total divided by the number of jets. Two types of manifold were tried. Linear (Fig. 2c) and radial (Fig. 2d). Results for both are plotted in Fig. 6; the linear manifold has either 8 or 22 jets and the radial has either 4 or 8 jets incorporated into the manifold. It is clear that a linear manifold yields much higher values of $k$ - hence greater mixing - than that of the standard single straight inlet. It is also clear from Fig. 6 that the value of $k$ is increased by subdivision into smaller diameter jets. However, the radial manifold is seen to produce results which are closely comparable with but not better than (for minimised mixing) the standard case, irrespective of the number of jets.

The final inflow consideration considered (Fig. 2e) has the inflow jet directed downwards. Both the jetorifice distance from the base, $y$, and side wall, $x$, were varied. However, it was found that the results for this case, presented in Fig. 6, were insensitive to $x$. (See the two sets of results for $y=5 \mathrm{~mm}$.) In contrast, values of the coefficient of mixing, $k$, decrease as $y$ is decreased. The gradients of the best-fit lines in Fig. 7 lead to the following approximation of $k$ upon $y$,

$$
k=\frac{1}{2}\left(\frac{y}{D}\right)+0.4 \text {. }
$$

Finally, the dependence of the coefficient of mixing upon inlet configuration is summarised in Table 1. Clearly a vertical inlet generates the least mixing of water already in the storage tank for a given inflow Froude number. However, it will be seen in the dimensioned applications, a radial manifold proves to be more practicable.

\section{APPLICATION TO ROOFTOP RAINWATER HARVESTING}

Having determined the coefficient of mixing for the various inlet arrangements shown in Fig. 2, we can now assess whether storage is a viable strategy for bacteria reduction in typical rainwater storage tanks used in developing countries. First we note that the volumetric inflow rate is given by $I_{R} A_{R} C_{R}$, where $I_{R}$ is the rainfall intensity, $A_{R}$ is the roof catchment area and $C_{R}$ is the run-off coefficient. The mean speed of the fluid jet(s) is thus given by

$$
u=\frac{I_{R} A_{R} C_{R}}{A_{j}}
$$


where $A_{j}$ is the (total) cross-sectional area of the jet(s). This, combined with (1)-(4), gives the dimensional value of the mixing height as

$$
h=\frac{129 I_{R} A_{R} C_{R} k}{\left(d^{3} T_{t} \Delta T\right)^{\frac{1}{2}}} \times \beta,
$$

for a single jet of diameter $d$; a similar expression can be found for the manifold arrangements. In (10), $\Delta T$ is the temperature difference between the water already resident $\left(\right.$ at $T_{t}^{\circ} \mathrm{C}$ ) in the tank and that of the inflow whilst the factor $\beta$ is given by

$$
\beta=\left[\frac{1-0.000005\left(T_{t}^{2}+T_{t}\right)}{1-(\Delta T-1) /\left(2 T_{t}\right)}\right]^{\frac{1}{2}}
$$

For $\Delta T=5^{\circ} \mathrm{C}$ and the range of applicability of (1), $\beta$ lies in the range $1.018-1.074$ and so can reasonably be approximated by unity.

We now evaluate (10) using data for two types of rainwater storage tanks found in developing countries, namely the Thai household rainwater jar and the tank used in the ALDEP catchment system from Botswana. From Gould \& Nissen-Petersen (1999), values of $A_{R}$, tank capacity and tank height, $H_{t}$ are included in Table 2. We use a typical temperature difference, $\Delta T=5^{\circ} \mathrm{C}$ and an ambient water temperature, $T_{t}=25^{\circ} \mathrm{C}$. Rainfall intensity, $I_{R}$, has been taken to be $4 \mathrm{~mm} /$ minute $\left(6.7 \times 10^{-5} \mathrm{~m} / \mathrm{s}\right)$, this high value occurring only for short periods and, in reality, often limited by gutter capacity. We have assumed that $C_{R}=1$. These values represent the worst case for reduced mixing. Table 2 then lists the predicted mixing heights, $h$, for a variety of inflow arrangements. Clearly, if $h \geq H_{t}$, then all the water already resident in the tank will be mixed with that of the roof water inflow and the strategy for bacteria reduction fails. Highlighted in Table 2 are those inlet arrangements which would result in $h<H_{t}$. This is the weakest criterion for a successful strategy. If the rainfall duration, $t_{R}$, is sufficiently long, then it can be seen from Fig. 3c that the height of mixed water, $h_{d}$ can reach the top of the tank, not through further mixing but through the continued addition of water after $t_{m}$. To preserve some unmixed water in the tank at the end of a single rainfall event of time $t_{R}$, the condition $h_{d}\left(t_{R}\right)<H_{t}$ must hold. This then requires that

$$
t_{R}<\frac{A_{t}\left(H_{t}-h\right)}{I_{R} A_{R} C_{R}},
$$

where $A_{t}$ is the cross-sectional area of the tank. If water is to spend sufficient time in the ageing zone over a succession of rainfall events, then $H_{t}$ in both (12) and the weaker condition, $h<H_{t}$, should be replaced by a suitable fraction of $H_{t}$. Since, for example, $h=0.42 H_{t}$ (Thai jar) or $0.09 H_{t}$ (ALDEP) for the radial manifold in Table 2, the proposed strategy for pathogen reduction through storage could be workable.

\section{CONCLUSIONS}

The effect of inlet configuration on the mixing of higher-density water and water already in an tank has been experimentally investigated. It has been shown that the relationship between a non-dimensional mixing height and inlet-jet densimetric Froude number, proposed by Fisher et al. (1979) and validated by Rossman \& Grayman (1999) for full mixing of a confined body of water, continues to hold for the zonal mixing herein and for a variety of both simple and geometrically complex jet arrangements. We have shown how the constant of proportionality - or mixing coefficient, $k$ - is dependent upon the inlet arrangement. For single jets, the lowest values of $k$ are found for a downward jet in close proximity to the tank base whilst for horizontal jets, those directed tangentially along the tank wall yield a lower $k$ than those radially directed from the tank wall. Multiple jets arranged in a linear manifold give higher $k$ values than simple jets whilst those for radial (outwardly from the tank centre) manifolds are comparable. Radial manifolds offer a means to increase the total crosssectional area of the inlet and may have the most utility in applications. Our investigation has led us to propose inlet configurations which will minimise the mixing in storage tanks, a desirable objective for the reduction of pathogens in rooftop rainwater harvesting systems.

\section{APPENDIX I. REFERENCES}

Davidson, J.H. and Adams, D.A. (1994) "Fabric stratification manifolds for solar water heating", ASME: Journal of Solar Energy Engineering, 116, 130-136.

Droste, R.L. (1997) Theory and practice of waste-water treatment, Wiley, New York.

Fischer, H. B., List, E. J., Kohl, R. C., Imberger, J. and Brookes, N. H. (1979). Mixing in inland and coastal waters, Academic Press, New York.

Gould, J. and Nissen-Petersen, E. (1999). Rainwater Catchment Systems for domestic supply, Intermediate Technology Publications, London.

Linden, P.F. (1999). "The fluid mechanics of natural ventilation", Annual Review of Fluid Mechanics, 21, 201-238.

Rossman and Grayman (1999). "Scale model studies of mixing in drinking water storage tanks", ASCE Journal of Environmental Engineering, 25, 755-761.

\section{APPENDIX II. NOTATION}

The following symbols are used in this paper:

$A_{t}=$ cross-sectional area of storage tank;

$A_{R}=$ roof area;

$A_{j}=$ (total) cross-sectional area of jet(s);

$C_{r}=$ run-off coefficient;

$d=$ diameter of incoming stream/inlet diameter;

$D=$ tank diameter;

$F_{d}=$ densimetric Froude number $\left[u /\left(g^{\prime} d\right)^{\frac{1}{2}}\right]$;

$g=$ acceleration due to gravity; 
$g^{\prime}=$ reduced gravity (defined in (2));

$h=$ vertical extent of mixed water in tank;

$h_{d}=$ height of mixed water above inlet;

$h_{w}=$ height of water surface above tank inlet;

$H_{t}=$ height of storage tank;

$I_{R}=$ rainfall intensity;

$k=$ constant - coefficient of mixing - defined by (4);

$t_{m}=$ mixing time;

$t_{f}=$ time (duration) of experimental run;

$t_{R}=$ time (duration) of rainfall event;

$T=$ temperature $\left(\right.$ in $\left.{ }^{\circ} \mathrm{C}\right)$;

$T_{t}=$ temperature of tank water;

$\Delta T=$ temperature difference between tank and inflow water;

$u=$ mean speed of inflow;

$x=$ distance from tank wall;

$y=$ distance from tank base;

$\rho=$ water density;

$\rho_{f}=$ density of inflow water;

$\rho_{t}=$ density of tank water;

$\beta=$ expression defined by (12).

\section{Subscripts}

$m=$ model;

$p=$ prototype;

$t=$ tank;

$R=$ rainfall; 